\title{
New metastable phases in an oxyborate compound obtained by an evolutionary algorithm and Density Functional Theory
}

\author{
E. Vallejo ${ }^{a, *}$ M. Avignon ${ }^{b}$ \\ ${ }^{a}$ Escuela Superior de Apan, Universidad Autónoma del Estado de Hidalgo. Carretera Apan-Calpulalpan Km. 8, Col. Chimalpa, C.P. 43920, \\ Apan, Hidalgo, México. \\ b Institut Néel, Centre National de la Recherche Scientifique (CNRS) and Université Joseph Fourier, BP 166, 38042 Grenoble Cedex 9, France
}

\begin{abstract}
New metastable phases in the Fe homometallic ludwigite compound are obtained and studied using an evolutionary algorithm and Density Functional Theory. Our lowest energy monoclinic structure is identified as $\mathrm{P} 22_{1} / \mathrm{m}$ with space group number of 11 . This structure evolves towards the monoclinic structure as the result of the spin orbit coupling and a particular zigzag magnetic structure. A zigzag distortion in a class of three-leg ladders follows similar to the experimental one observed below the transition temperature of $\mathrm{T}_{c}=283 \mathrm{~K}$. In this distortion long and short bonds inside rungs alternating in a zigzag way along the ladder legs. Furthermore, a new type of zigzag structural ordering is observed in other two low-energy phases analyzed. In this case, the magnetic ordering behaves qualitatively similar to the experimental structure at $82 \mathrm{~K}$, with antiferromagnetically coupled ferromagnetic rungs. Our calculations show that magnetic symmetry is not favorable for zigzag structural ordering. Finally, structural and magnetic properties will be discussed in comparison with the experimentally known phases.
\end{abstract}

Key words: Ab initio calculations (electronic structure of atoms and molecules), Strongly correlated electron systems; heavy fermions, Magnetism and magnetic materials systems.

PACS: 31.15.A-, 31.15.E-, 71.27.+a

\section{Introduction}

Exciting properties like structural, transport and magnetic ones have been studied in a class of oxyborates known as warwickites [1-3] and ludwigites [4-10]. Interesting physical behavior like Wigner glass, the existence of a weak ferromagnetism or even charge ordering has been observed in ludwigite materials as consequence of strong correlations and low dimensional effects. Warwickites are characterized by one-dimensional structures called ribbons where the transition metals are randomly located $[1,2]$. Ludwigites in contrast, have a crystalline structure that consists of an assembling of subunits in the form of zigzag walls, with four nonequivalent octahedral sites occupied by divalent o trivalent metallic ions [5]. Warwickites $(q=1)$ and ludwigites $(q=2)$ present the following chemical formula $\mathrm{M}_{q} \mathrm{O}_{q} \mathrm{M}^{\prime} \mathrm{BO}_{3}$ where $\mathrm{M}\left(\mathrm{M}^{\prime}\right)$ are divalent (trivalent) $3 \mathrm{~d}$ transition-metal ions.

\footnotetext{
* Corresponding author.

Email address: emapion2@gmail.com (E. Vallejo).
}

Among the ludwigites, the semiconductor homometallic $\left(\mathrm{M}=\mathrm{M}^{\prime}=\mathrm{Fe}\right) \mathrm{Fe}_{3} \mathrm{O}_{2} \mathrm{BO}_{3}$ is particularly interesting, not only because of its intrinsic properties but also because of the underlying physics of spin ladders. Its structure can be viewed as formed of two magnetic subsystems which can be considered as decoupled to a first approximation [5]. Each consists of three-leg spin ladders subunits of $\mathrm{Fe}^{2+}$ and $\mathrm{Fe}^{3+}$ cations. The first type consists of triads (Fe4-Fe2-Fe4) of $\mathrm{Fe}^{3+}$ ions with one itinerant electron per rung, while the second one is formed by triads (Fe3-Fe1-Fe3) of $\mathrm{Fe}^{2+}$ ions. These three-leg ladders are present in our structures in figures 1 and 2 and clearly evidenced in figures 3 and 4 for the 1_O phase corresponding to Fig. 2. A perspective in the a-b (x-y) plane of the Fe4-Fe2-Fe4 triads can be oberved in Fig. 3. In the same figure three near diagonal Fe (blue) atoms form the triads. In figure 3 can be observed that $\mathrm{Fe} 4-\mathrm{Fe} 2-$ $\mathrm{Fe} 4$ triads share the same plane with the group $\left(\mathrm{BO}_{3}\right)^{-3}$. Fe3-Fe1-Fe3 triads are shown in figure 4 as three near horizontal Fe atoms. Fe4-Fe2-Fe4 ladders exhibit charge ordering and structural phase transition at $\mathrm{T}_{c}=283 \mathrm{~K}$ observed using specific-heat measurements [6] and X-ray diffraction [7]. This transition is accompanied by a change in the ac- 
tivation energy of the electrical resistivity [7]. Below $\mathrm{T}_{c}$, each triad presents a short and long bond alternating along the ladder c-axis [7] giving a zigzag structural arrangement similar to the one presented in Fig 5-a). The overall crystal structure is orthorhombic, space group Pbnm. In the high temperature phase all bonds become equal in the triads as can be observed in figure 5-c) and the symmetry is orthorhombic, space group Pbam.

An antiferromagnetic transition at $112 \mathrm{~K}$ has been observed. This transition basically involves $\mathrm{Fe}^{3+}$ and $\mathrm{Fe}^{2+}$ within the first magnetic system $[11,12]$. The complementary Fe-ions are paramagnetic down to $74 \mathrm{~K}$. Below $70 \mathrm{~K}$ all the sample becomes magnetically ordered. Mostly $\mathrm{Fe}^{3+}$ ions order in a weak ferromagnetism where canting of the magnetic hyperfine field for each Fe-ion is related to this order $[5,6,12]$. A total antiferromagnetic state is found below $\sim 40 \mathrm{~K}$ for this Fe-ludwigite. Below 40K, two-dimensional antiferromagnetic magnons were proposed to explain the low-temperature $\mathrm{T}^{2}$ behavior and disorder $\left(\mathrm{Fe}^{3+}-\mathrm{Fe}^{2+}\right)$ was proposed to explain the large linear term of the specific heat in $\mathrm{Fe}_{3} \mathrm{O}_{2} \mathrm{BO}_{3}$ [6] above $112 \mathrm{~K}$. Transport measurements in Fe-Ludwigite show activated behavior with two characteristic energies above and below charge ordering at 220K [5]. Theoretical investigation of spin exchange interactions and electronic structure were done using a spin dimer analysis [13] and the extended Hückel method [14] respectively. In contrast, an excitonic instability using a tight-binding model was studied in a three-leg ladder [15]. Nevertheless the important electronic, structural and magnetic correlation was taken into account by Vallejo and Avignon [16]. Spin and charge ordering was obtained resulting from the competition between double exchange and superexchange interactions. These interactions were proposed as a possible principal mechanism to study Ludwigites [16]. Double exchange interactions favor a ferromagnetic strong and short distance background of local spins. In contrast, superexchange interactions are responsible for antiferromagnetic ordering leading to interesting and unusual magnetic states [16]. Some of these magnetic phases obtained by Vallejo and Avignon are similar to these proposed to explain neutron powder diffraction experiments [11]. In the same way, similar magnetic behavior was found in a further theoretical work using density functional theory [17].

Contrastingly, the crystalline structure, space group and lattice parameters of the only other known homometallic oxyborate Co-based ludwigite $\mathrm{Co}_{3} \mathrm{O}_{2} \mathrm{BO}_{3}$ are very similar to the previous Fe one at room temperature. These similarities suggest similar behaviors, nevertheless their physical properties are very distinct [18]. Structural distortion and two magnetic transitions $(\simeq 112 \mathrm{~K}$ and $\simeq 70 \mathrm{~K})$ are not found for the Co ludwigite [18]. Only one antiferromagnetic transition at $42 \mathrm{~K}$ is found for the former material [18]. In this point, it is interesting to know the reason for the different physical behavior of these ludwigites. Spin-orbit coupling was proposed as a detriment to the Peierls transition, inhibiting the structural transition for the Co-Ludwigite [18]. However, it was proposed that orbital moments of Co ions are almost quenched [9]. For this reason, using the extra electron for the Co a disorder-based mechanism was discused in reference [19].

In this paper we focus on structural and magnetic properties of a particular oxyborate compound known as Ludwigite using an evolutionary algorithm and Density Functional Theory. An orthorhombic-monoclinic structural transition was observed where spin-orbit coupling and a particular magnetic structure were proposed as an explanation of this transition. The structural and magnetic properties of this monoclinic phase follows a zigzag distortion like the zigzag structural one observed in the Fe-homometallic Ludwigite compound. These structural and magnetic properties will be discussed in comparison with the experimentally observed phases in the Fe-Ludwigite system.

The paper is organized as follows. In section 2 a brief description of the computational methods is given. In section 3 , results and a discussion are presented. Finally, our results are summarized in section 4 .

\section{Computational methods}

In this work, an evolutionary algorithm as implemented in The Universal Structure Predictor: Evolutionary Xtallography (USPEX) code [20-22] and the Vienna Ab-initio Simulation Package (VASP) $[23,24]$ were used to obtain several $\mathrm{Fe}_{3} \mathrm{O}_{2} \mathrm{BO}_{3}$ Ludwigite low-energy metastable phases. Recently, the former evolutionary algorithm has been used to study new reconstructions of the (110) surface of Rutile $\mathrm{TiO}_{2}$ [25] for the design of organic polymer dielectrics [26] and to obtain new metastable phases in a trititanium pentoxide compound [27]. Density Functional Theory (DFT), the Projector Augmented Wave (PAW) method [28,29] besides the GGA functionals were also utilized. Additionally the exchange correlation form according to the PerdewBurke-Ernzerhof (PBE) [30] exchange correlation was considered as used in ref. [17]. Collinear and non-collinear (including spin-orbit interaction) spin polarization calculations and the on-site Coulomb interaction within the Dudarev's Approach [31] were utilized to take into account the highly correlated characteristics of $\mathrm{Fe}_{3} \mathrm{O}_{2} \mathrm{BO}_{3}$ Ludwigite. In this work it was chosen $\mathrm{J}=0.5 \mathrm{eV}$ with $\mathrm{U}=5.5 \mathrm{eV}$ being $\mathrm{U}-\mathrm{J}=5.0 \mathrm{eV}$. On the other hand, Monkhorst-Pack sampling and a value of $520 \mathrm{eV}$ for the energy cutoff were used. In all our calculations the $3 \mathrm{p} 3 \mathrm{~d} 4 \mathrm{~s}, 2 \mathrm{~s} 2 \mathrm{p}$ and $2 \mathrm{~s} 2 \mathrm{p}$ configurations were considered as valence electrons for $\mathrm{Fe}, \mathrm{B}$ and $\mathrm{O}$ atoms respectively. Finally, an exhausted analysis was carefully performed to ensure convergence in k-points $(3 \times 3 \times 6$ and $4 \times 4 \times 8$ ), electronic charge density difference (less than $1 \times 10^{-4}$ ) and in the ionic force convergence criterion (less than $\left.1 \times 10^{-2} \mathrm{eV} / \AA\right)$.

Inside the evolutionary algorithm, it is important to mention that softmutate variation operators were mostly utilized each generation $(>50 \%)$ and are responsible for the most important phases presented in this work. Softmutation creates new structures by large displacements of the 
atoms along the eigenvectors of the soft phonon modes [20$22]$. In contrast, enthalpy was the property to optimize.

\section{Results and discussions}

The main result of this work is presented in Table 1. Several low-energy metastable phases were obtained from total free energy calculations after variable (or static) cell and atomic position optimization. M. Matos and co-workers [17] studied the magnetic behavior of this Fe-Ludwigite using static atomic positions and lattice parameters calculations. In our work, instead of results of M. Matos, We observe new phases having lower energy after full dynamic variable optimization. For example, 4_O_NC (2_O_NC) phases were obtained using a DFT static (dynamic) ionic and cell relaxation with Pbnm experimental structure as seed, as proposed by M. Matos. It is clear (see Table 1) that there is a considerable reduction of energy using a dynamic relaxation. Furthermore, most of our phases presented in Table 1 were obtained using the evolutionary algorithm for example the (5_T and 10_T) triclinic phases.

Lattice parameters and space groups together with volume and a relative energy $\Delta(E)$ defined as $\mathrm{E}$ (phase)E(1_M_NC phase) are also shown in Table 1. In this way, collinear and non-collinear (including spin-orbit interaction) spin polarization calculations were performed, and several different magnetic configurations, were proposed and tested as the ground state. The Pbam and Pbnm (see Table 1) experimental phases were proposed as the initial structural seed to start with the calculations. For example 4_O_NC (2_O_NC) phases were obtained using a DFT static (dynamic) ionic relaxation with Pbnm experimental structure as seed. After relaxation we observed an increase of lattice parameters and a significant reduction of total energy in the case of the 2_O_NC phase as indicated previously.

Our discussions in this paper will be focused on phases that present $\Delta(E) \leq 25 \mathrm{meV}$ ( $25 \mathrm{meV}$ corresponds to thermal energy at $20^{\circ} \mathrm{C}$ ). For example 1_M_NC and 2_M phases were optimized using different initial magnetic configurations and the initial orthorhombic Pbam experimental crystal structure as seed. In contrast, 1_O was optimized using the initial Pbnm crystal structure. Our lowest energy monoclinic 1_M_NC lattice system is a new metastable phase obtained in the $\mathrm{Fe}_{3} \mathrm{O}_{2} \mathrm{BO}_{3}$ Ludwigite. This novel result is the important contribution of this work.

In a general way, for the 1_M_NC and 2_M structures (Figure 1) and 1_O structure (Figure 2) we present selected Fe-Fe distances in Table 2, as well as site-magnetization and total and partial charge in Table 3.

\subsection{The 1_O and 2_M structures}

A qualitative picture of the orthorhombic 1_O and monoclinic 2_M structures, can be observed in figures 2 and 1 respectively. The 1_O and 2_M structures were identi-

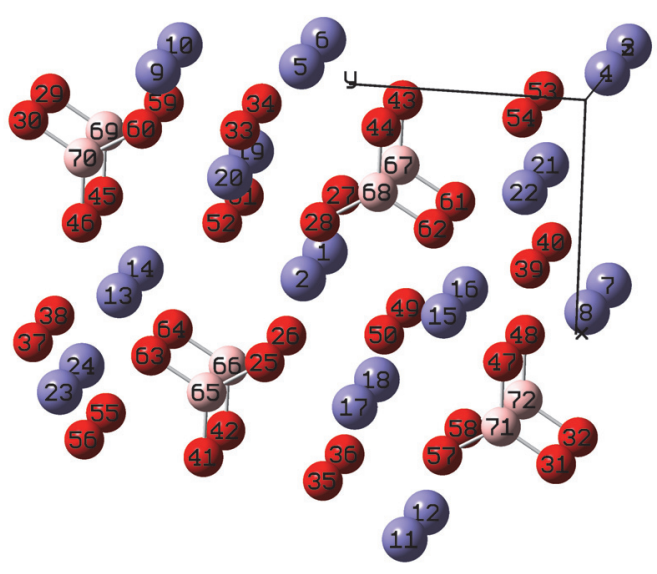

Fig. 1. Perspective of the 3D (three dimensional) unit cell of the 1_M_NC and 2_M structures. Blue, red and pink color corresponds to $\mathrm{Fe}, \mathrm{O}$ and $\mathrm{B}$ atoms respectively. Numbering was included to identify atoms.

fied as $\mathrm{Pmn} 21$ and $\mathrm{P} 2 / \mathrm{m}$ with space group numbers of $\left(\mathrm{N}^{o}\right.$ $31)$ and $\left(\mathrm{N}^{o} 10\right)$ respectively. FINDSYM program was used for identifying the space-group symmetry [32]. Furthermore it is important to mention that a tolerance of $1 \times 10^{-4}$ was used to identify space groups. We realise that the orthorhombic experimental Pbam ( $\left.\mathrm{N}^{o} 55\right)$ and $\mathrm{Pbnm}\left(\mathrm{N}^{o} 62\right)$ phases have higher symmetry than the former phases presented. The former comparison is important because 1_O and 2_M structures were optimized using the initial Pbnm and Pbam experimental (respectively) crystal structures with high symmetry. The initial magnetic structure consists of ferromagnetic rungs coupled antiferromagnetically along c-axis for the Fe4-Fe2-Fe4 ladders and antiferromagnetic rungs coupled ferromagnetically along c-axis for the Fe3-Fe1-Fe3 ladders as can be oberved in Fig. 5 .

In $\mathrm{Fe} 4-\mathrm{Fe} 2-\mathrm{Fe} 4$ ladders, a new zigzag structural ordering results in both systems as presented in Figure 5-b). Large and short rungs intercalate in a zigzag way along c direction. The Fe4-Fe2 distance for the short rung varies from $2.836 \AA$ (in the case of the 2_M structure) to $2.839 \AA$ (in the case of the 1_O structure), see Table 2 . In contrast, an increase $(0.013 \AA)$ to $2.849 \AA$ (in the case of the 2_M structure) and (0.007 $\AA$ ) $2.846 \AA$ (in the case of the 1_O structure) was calculated for large rungs. Magnetic (Ferromagnetic) symmetry within the rungs does not favor different bond lengths and is the source of the low rise between long and short rungs distances. In the case of 1_O structure, it is clear that the initial zigzag ordering of Pbnm structure 
Table 1

Collinear and non-collinear low-energy metastable phases. Lattice parameters (a, b and c in $\AA$ ) and angles $\left(\alpha, \beta\right.$ and $\gamma$ in $\left({ }^{\circ}\right)$ ). Crystal volume (in $\AA^{3}$ ) and a relative energy $\Delta(E)$ (in eV) are also shown. Asterisk (*) indicates that is not a primitive cell. O, M, T and NC stands for orthorhombic, monoclinic, triclinic and non-collinear lattice systems respectively. For details see text.

\begin{tabular}{|c|c|c|c|c|c|c|c|c|c|c|}
\hline Crystal system & Space group & No. & $\mathrm{a}$ & $\mathrm{b}$ & $\mathrm{c}$ & Volume & $\alpha$ & $\beta$ & $\gamma$ & $\Delta(E)$ \\
\hline \multicolumn{11}{|l|}{ Collinear phases } \\
\hline $1 \_\mathrm{O}$ & $\mathrm{Pmn} 2_{1}$ & 31 & 9.608 & 12.452 & 6.206 & 742.489 & 90.000 & 90.000 & 90.000 & 0.016 \\
\hline 2_M & $\mathrm{P} 2 / \mathrm{m}$ & 10 & 9.605 & 12.455 & 6.208 & 742.736 & 90.000 & 90.000 & 89.999 & 0.018 \\
\hline $3 \_\mathrm{O}$ & $\mathrm{P} 2{ }_{1} 2_{1} 2$ & 18 & 9.462 & 12.308 & 6.150 & 716.219 & 90.000 & 90.000 & 90.000 & 0.533 \\
\hline $4 \_\mathrm{O}$ & $\mathrm{Pmn} 2_{1}$ & 31 & 9.449 & 12.282 & 6.150 & 713.724 & 90.000 & 90.000 & 90.000 & 0.640 \\
\hline 5_T & $\mathrm{P} 1$ & 1 & 9.636 & 12.491 & 6.206 & 746.980 & 89.912 & 90.008 & 90.006 & 1.204 \\
\hline 6_O* & Pbam & 55 & 9.636 & 12.486 & 6.202 & 746.199 & 90.000 & 90.000 & 90.000 & 1.252 \\
\hline 7_O & Pnma & 62 & 9.635 & 12.488 & 6.202 & 746.156 & 90.000 & 90.000 & 90.000 & 1.258 \\
\hline 8_O* & Pbam & 55 & 9.462 & 12.308 & 6.150 & 716.219 & 90.000 & 90.000 & 90.000 & 1.940 \\
\hline 9_O & Pnma & 62 & 9.449 & 12.282 & 6.150 & 713.724 & 90.000 & 90.000 & 90.000 & 2.078 \\
\hline $10 \_\mathrm{T}$ & $\mathrm{P} 1$ & 1 & 9.605 & 12.461 & 6.185 & 740.240 & 89.744 & 89.729 & 90.022 & 2.271 \\
\hline \multicolumn{11}{|c|}{ Non-collinear phases } \\
\hline 1_M_NC & $\mathrm{P} 2{ }_{1} / \mathrm{m}$ & 11 & 9.620 & 12.448 & 6.211 & 743.740 & 90.000 & 90.000 & 90.004 & 0.000 \\
\hline $2 \_\mathrm{O} \_\mathrm{NC}$ & Pnma & 62 & 9.609 & 12.456 & 6.202 & 742.280 & 90.000 & 90.000 & 90.000 & 0.026 \\
\hline 3_O_NC & $\mathrm{Pmn} 2_{1}$ & 31 & 9.623 & 12.448 & 6.212 & 744.150 & 90.000 & 90.000 & 90.000 & 0.034 \\
\hline 4_O_NC & Pnma & 62 & 9.449 & 12.282 & 6.150 & 713.720 & 90.000 & 90.000 & 90.000 & 24.692 \\
\hline \multicolumn{11}{|c|}{ Experimental phases } \\
\hline $\operatorname{Exp}[7]$ & Pbam & 55 & 9.462 & 12.308 & 3.075 & 358.112 & 90.000 & 90.000 & 90.000 & \\
\hline $\operatorname{Exp}[7]$ & Pbnm & 62 & 9.449 & 12.282 & 6.150 & 713.763 & 90.000 & 90.000 & 90.000 & \\
\hline
\end{tabular}

Table 2

Selected Fe-Fe distances (in $\AA$ ) of the 1_O, 2_M and 1_M_NC $\mathrm{Fe}_{3} \mathrm{O}_{2} \mathrm{BO}_{3}$ Ludwigite metastable phases and compared with the experimental (Exp) ones of the Fe4-Fe2-Fe4 ladders, see figures 1 and 2 as references. Asterisk (*) implies crystallographically equivalent atoms in Figs. 1 and 2. Details in text.

\begin{tabular}{lllll}
$1 \_\mathrm{O}$ & 2_M & 1_M_NC & Pbam Exp & Pbnm Exp \\
\hline
\end{tabular}

\begin{tabular}{|c|c|c|c|c|c|c|}
\hline \multicolumn{7}{|c|}{ Fe4-Fe2-Fe4 ladders [7] } \\
\hline $\mathrm{Fe}(20)-\mathrm{Fe}(8)$ & 2.846 & $\mathrm{Fe}\left(23^{*}\right)-\mathrm{Fe}(8)$ & 2.836 & 2.885 & $2.786[7]$ & $2.942[7]$ \\
\hline $\mathrm{Fe}(8)-\mathrm{Fe}\left(24^{*}\right)$ & 2.846 & $\mathrm{Fe}(8)-\mathrm{Fe}(22)$ & 2.836 & 2.796 & $2.786[7]$ & $2.616[7]$ \\
\hline $\mathrm{Fe}(23)-\mathrm{Fe}(7)$ & 2.839 & $\mathrm{Fe}\left(24^{*}\right)-\mathrm{Fe}(7)$ & 2.849 & 2.796 & $2.786[7]$ & $2.616[7]$ \\
\hline $\mathrm{Fe}(7)-\mathrm{Fe}\left(19^{*}\right)$ & 2.839 & $\mathrm{Fe}(7)-\mathrm{Fe}(21)$ & 2.849 & 2.885 & $2.786[7]$ & $2.942[7]$ \\
\hline $\mathrm{Fe}(20)-\mathrm{Fe}(23)$ & 3.103 & $\mathrm{Fe}\left(23^{*}\right)-\mathrm{Fe}\left(24^{*}\right)$ & 3.104 & 3.106 & $3.075[7]$ & $3.075[7]$ \\
\hline $\mathrm{Fe}(8)-\mathrm{Fe}(7)$ & 3.103 & $\mathrm{Fe}(8)-\mathrm{Fe}(7)$ & 3.104 & 3.107 & $3.075[7]$ & $3.075[7]$ \\
\hline $\mathrm{Fe}\left(24^{*}\right)-\mathrm{Fe}\left(19^{*}\right)$ & 3.103 & $\mathrm{Fe}(22)-\mathrm{Fe}(21)$ & 3.104 & 3.106 & $3.075[7]$ & $3.075[7]$ \\
\hline \multicolumn{7}{|c|}{ Fe3-Fe1-Fe3 ladders [7] } \\
\hline $\mathrm{Fe}(2)-\mathrm{Fe}(15)$ & 3.425 & $\mathrm{Fe}(2)-\mathrm{Fe}(16)$ & 3.426 & 3.427 & & \\
\hline $\mathrm{Fe}(2)-\mathrm{Fe}(16)$ & 3.425 & $\mathrm{Fe}(2)-\mathrm{Fe}(14)$ & 3.426 & 3.427 & & \\
\hline $\mathrm{Fe}\left(1^{*}\right)-\mathrm{Fe}(13)$ & 3.425 & $\mathrm{Fe}\left(1^{*}\right)-\mathrm{Fe}(13)$ & 3.426 & 3.427 & & \\
\hline $\mathrm{Fe}\left(1^{*}\right)-\mathrm{Fe}(14)$ & 3.425 & $\mathrm{Fe}\left(1^{*}\right)-\mathrm{Fe}(15)$ & 3.426 & 3.427 & & \\
\hline $\mathrm{Fe}(13)-\mathrm{Fe}(16)$ & 3.099 & $\mathrm{Fe}(13)-\mathrm{Fe}(14)$ & 3.103 & 3.114 & & \\
\hline $\mathrm{Fe}(2)-\mathrm{Fe}\left(1^{*}\right)$ & 3.105 & $\mathrm{Fe}(2)-\mathrm{Fe}\left(1^{*}\right)$ & 3.114 & 3.106 & & \\
\hline $\mathrm{Fe}(14)-\mathrm{Fe}(15)$ & 3.099 & $\mathrm{Fe}(15)-\mathrm{Fe}(16)$ & 3.103 & 3.098 & & \\
\hline
\end{tabular}


Table 3

Selected site-magnetization (m) (left) (in Bohr magnetons) and total charge (q) (right) of the $\mathrm{Fe}_{3} \mathrm{O}_{2} \mathrm{BO}_{3} \mathrm{Ludwigite}$ metastable phases, see figures 1 and 2. d-orbitals partial charge $\left(\mathrm{q}_{d}\right)$ for $1 \_\mathrm{O}$ and 2_M phases was included as reference. Details in text.

\begin{tabular}{|c|c|c|c|c|c|c|c|c|c|c|}
\hline Atoms & $1 \_\mathrm{O}$ & & & 2_M & & & M_NC & & & \\
\hline & $\mathrm{m}_{z}$ & $q$ & $\mathrm{q}_{d}$ & $\mathrm{~m}_{z}$ & $q$ & $\mathrm{q}_{d}$ & $\mathrm{~m}_{x}$ & $\mathrm{~m}_{y}$ & $\mathrm{~m}_{z}$ & $q$ \\
\hline $\mathrm{Fe}(1)$ & -3.724 & 12.379 & 5.949 & 3.727 & 12.378 & 5.948 & 0.009 & -0.015 & -3.725 & 12.377 \\
\hline $\mathrm{Fe}(2)$ & -3.724 & 12.379 & 5.949 & 3.727 & 12.378 & 5.948 & -0.009 & 0.015 & -3.725 & 12.377 \\
\hline $\mathrm{Fe}(3)$ & -3.724 & 12.379 & 5.949 & -3.727 & 12.378 & 5.948 & 0.014 & 0.013 & 3.726 & 12.377 \\
\hline $\mathrm{Fe}(4)$ & -3.724 & 12.379 & 5.949 & -3.727 & 12.378 & 5.948 & -0.014 & -0.012 & 3.726 & 12.377 \\
\hline $\mathrm{Fe}(5)$ & 3.752 & 12.390 & 5.920 & -3.745 & 12.391 & 5.922 & -0.000 & -0.000 & 3.757 & 12.386 \\
\hline $\mathrm{Fe}(6)$ & -3.736 & 12.392 & 5.925 & 3.743 & 12.390 & 5.923 & -0.000 & -0.000 & 3.757 & 12.386 \\
\hline $\mathrm{Fe}(7)$ & 3.752 & 12.390 & 5.920 & -3.743 & 12.390 & 5.923 & -0.000 & -0.000 & 3.757 & 12.387 \\
\hline $\mathrm{Fe}(8)$ & -3.736 & 12.392 & 5.925 & 3.745 & 12.391 & 5.922 & -0.000 & -0.000 & 3.757 & 12.387 \\
\hline $\mathrm{Fe}(9)$ & 3.723 & 12.392 & 5.953 & 3.723 & 12.391 & 5.953 & 0.007 & 0.000 & -3.719 & 12.392 \\
\hline $\mathrm{Fe}(10)$ & 3.723 & 12.392 & 5.953 & 3.723 & 12.391 & 5.953 & -0.007 & -0.000 & -3.720 & 12.391 \\
\hline $\mathrm{Fe}(11)$ & 3.723 & 12.392 & 5.953 & 3.723 & 12.391 & 5.953 & 0.007 & 0.000 & -3.720 & 12.391 \\
\hline $\mathrm{Fe}(12)$ & 3.723 & 12.392 & 5.953 & 3.723 & 12.391 & 5.953 & -0.007 & -0.000 & -3.719 & 12.392 \\
\hline $\mathrm{Fe}(13)$ & 3.723 & 12.392 & 5.953 & -3.723 & 12.391 & 5.953 & 0.016 & 0.011 & 3.721 & 12.393 \\
\hline $\mathrm{Fe}(14)$ & 3.723 & 12.392 & 5.953 & -3.723 & 12.391 & 5.953 & -0.016 & -0.011 & 3.721 & 12.393 \\
\hline $\mathrm{Fe}(15)$ & 3.723 & 12.392 & 5.953 & -3.723 & 12.391 & 5.953 & 0.016 & 0.011 & 3.721 & 12.393 \\
\hline $\mathrm{Fe}(16)$ & 3.723 & 12.392 & 5.953 & -3.723 & 12.391 & 5.953 & -0.016 & -0.011 & 3.721 & 12.393 \\
\hline $\mathrm{Fe}(17)$ & 4.269 & 12.210 & 5.593 & -4.262 & 12.213 & 5.596 & 0.000 & -0.000 & 4.277 & 12.207 \\
\hline $\mathrm{Fe}(18)$ & -4.252 & 12.220 & 5.603 & 4.258 & 12.216 & 5.599 & -0.000 & 0.000 & -4.269 & 12.209 \\
\hline $\mathrm{Fe}(19)$ & 4.269 & 12.210 & 5.593 & 4.258 & 12.216 & 5.599 & -0.000 & 0.000 & 4.277 & 12.207 \\
\hline $\mathrm{Fe}(20)$ & -4.252 & 12.220 & 5.603 & -4.262 & 12.213 & 5.596 & 0.000 & -0.000 & -4.269 & 12.209 \\
\hline $\mathrm{Fe}(21)$ & 4.269 & 12.210 & 5.593 & -4.258 & 12.217 & 5.599 & 0.000 & -0.000 & -4.273 & 12.208 \\
\hline $\mathrm{Fe}(22)$ & -4.252 & 12.220 & 5.603 & 4.262 & 12.213 & 5.596 & -0.000 & -0.000 & 4.273 & 12.208 \\
\hline $\mathrm{Fe}(23)$ & 4.269 & 12.210 & 5.593 & 4.262 & 12.213 & 5.596 & -0.000 & -0.000 & -4.273 & 12.208 \\
\hline $\mathrm{Fe}(24)$ & -4.252 & 12.221 & 5.603 & -4.258 & 12.217 & 5.599 & -0.000 & 0.000 & 4.273 & 12.208 \\
\hline
\end{tabular}

is not kept.

The former structural ordering is very different from the one observed in the Pbnm experimental structure where triads have a slight zigzag appearance in the low temperature $(<283 \mathrm{~K})$ structure due to the small displacement of the central ions [7], to have an idea of the former structure see Figure 5-a). A possible explanation of this particular zigzag behavior (because of rungs distance) could be the well known structural instability in low dimensional systems. Contrastingly, structural and magnetic symmetry is observed in Fe3-Fe1-Fe3 ladders where a distance Fe3-Fe1 of $3.425 \pm 0.001$ Åwas calculated for both systems, see Table 2 .

Magnetic ordering in $\mathrm{Fe} 4-\mathrm{Fe} 2-\mathrm{Fe} 4$ ladders behave similarly to the Pbnm experimental structure at $82 \mathrm{~K}$, with ferromagnetic ordering inside rungs together with rungs ordered antiferromagnetically in c direction, see Figure 5-b) [11]. This magnetic phase corresponds to the $\mathrm{A}_{I}$ magnetic phase as proposed in ref [16]. Vallejo and Avignon obtained this phase using the double and superexchange mechanism in a tight-binding model. Furthermore it is observed that charge distribution is mostly located in the center of the triads (with about 6 electrons in d orbitals) in both structures, see $\mathrm{q}_{d}$ in Table 3. The magnetic moment in the center corresponds to $\sim 3.7$ Bohr magnetons $\left(\mu_{B}\right)$ that is very near to $4 \mu_{B}$ for high spin $\mathrm{Fe}^{2+}$ ions. In the extremes of the ladder $\left(\sim 5.6\right.$ electrons in d orbitals, see $\mathrm{q}_{d}$ in Table 3$)$ a magnetic moment of $4.2 \mu_{B}$ was obtained. Experimentally it was proposed that the $\mathrm{Fe} 4-\mathrm{Fe} 2-\mathrm{Fe} 4$ ladders are constituted by three $\mathrm{Fe}^{3+}$ ions and one itinerant electron. In our structures we observed nevertheless that the ladders could be formed of $\mathrm{Fe}^{3+}$ ions with two itinerant electrons. Contrastingly, the magnetic moments obtained by powder diffraction study [11] are $3.9 \mu_{B}$ (in the center) and $2.74 \mu_{B}$ (in the extremes). The former experimental results suggest that charge distribution is mostly located in the extremes of the ladder.

The magnetic behavior of Fe3-Fe1-Fe3 ladders was a little bit different as observed in the former ladders. We think that the large distance $(\sim 3.425 \AA$ Asee Table 2$)$ between ions decrease direct covering favoring antiferromagnetic behavior inside rungs. The magnetic behavior is qualitatively 

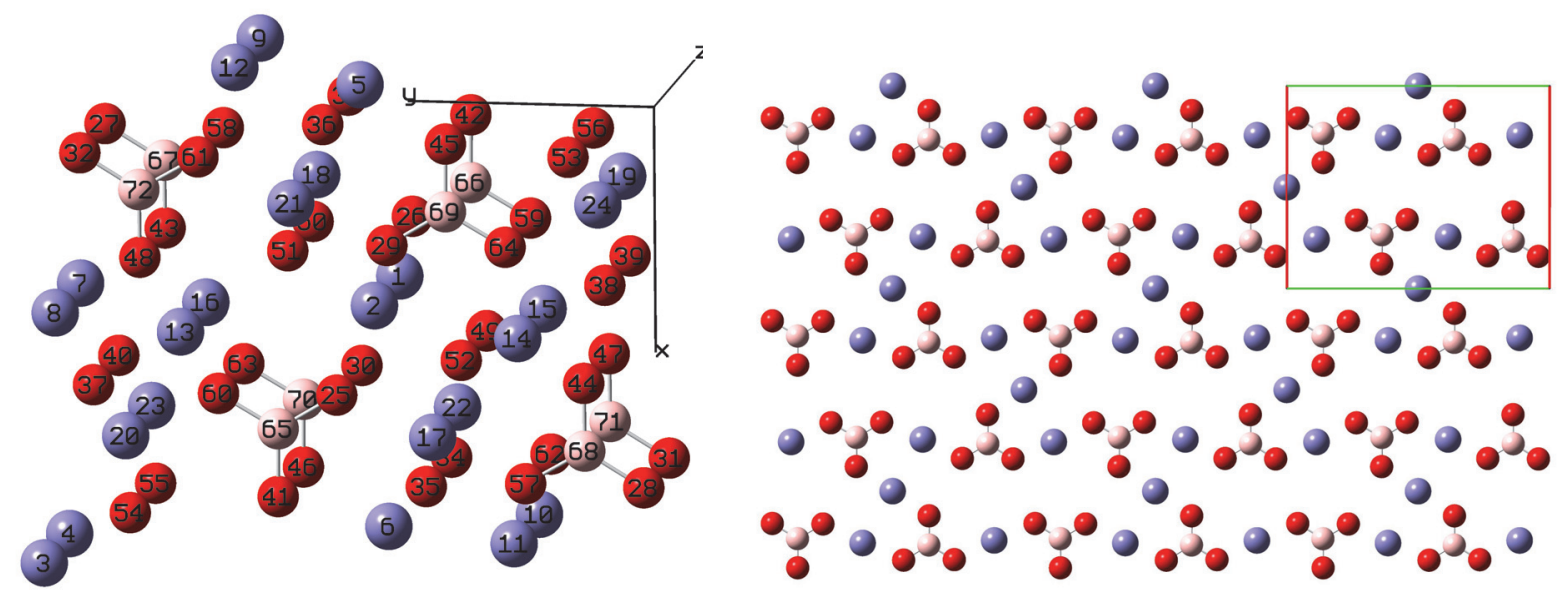

Fig. 2. Perspective of the 3D unit cell of the 1_O structure. Blue, red and pink color corresponds to $\mathrm{Fe}, \mathrm{O}$ and $\mathrm{B}$ atoms respectively. Numbering was included to identify atoms.

similar to the Pbnm experimental phase at $10 \mathrm{~K}$, with ferromagnetic chains along $\mathrm{c}$ direction coupled antiferromagnetically inside the rungs as presented in Figure 5-c) [11].

There is a charge $\left(\sim 6\right.$ electrons in d orbitals, see $\mathrm{q}_{d}$ in Table 3$)$ and magnetic moment $\left(\sim 3.7 \mu_{B}\right)$ symmetry in all ions in these ladders. In this case these ladders could be studied as high spin $\mathrm{Fe}^{2+}$ ions as experimentally proposed. Collinear spin polarised calculations were performed for both systems. It means that the quantization axis of spin for both $\mathrm{Fe} 4-\mathrm{Fe} 2-\mathrm{Fe} 4$ and $\mathrm{Fe} 3-\mathrm{Fe} 1-\mathrm{Fe} 3$ ladders is the same. The experimental (Pbnm structure) magnetic moments of these ladders are located in a perpendicular direction as compared with the direction of the Fe4-Fe2-Fe4 magnetic moments [11]. The experimental magnetic moments are 3.2 $\mu_{B}$ (in the center) and $4.0 \mu_{B}$ (in the extremes).

It is found that magnetic symmetry like the one observed in our 1_O and 2_M systems or even in the low temperature Pbnm experimental structure does not favor a zigzag structural ordering.

\subsection{The 1_M_NC structure}

A picture of the 1_M_NC structure is presented in figure 1. The 1_M_NC monoclinic structure was identified as $\mathrm{P} 2{ }_{1} / \mathrm{m}$ with space group number of $\left(\mathrm{N}^{o} 11\right)$ very near to the space group of ( $\left.\mathrm{N}^{\circ} 10\right)$ of the 2_M structure. It is important to mention that 1_M_NC structure was optimized using Pbam ( $\left.\mathrm{N}^{\circ} 55\right)$ experimental phase as the initial struc-
Fig. 3. Perspective in the a-b (x-y) plane at $\mathrm{z}=\mathrm{c} \sim 1.5 \AA$ of the $\mathrm{Fe} 4-\mathrm{Fe} 2-\mathrm{Fe} 4$ triads (three near diagonal Fe (blue) atoms) of the 1_O structure obtained from Fig. 2. Blue, red and pink color corresponds to $\mathrm{Fe}, \mathrm{O}$ and $\mathrm{B}$ atoms respectively. Rectangle is $\mathrm{a}=9.608 \AA$ (red line) and $b=12.452 \AA$ (green line) unit cell in accord to Table 1, and Fig. 2. Details in text.

ture. The Pbam phase is the high $(>283 \mathrm{~K}$ ) temperature phase without charge and structural distortion observed in the Fe Ludwigite. Non collinear spin polarised calculations together with spin-orbital coupling were used in our simulations.

We consider an initial magnetic phase corresponding to a zigzag ordering with one ferromagnetic and one antiferromagnetic bond within each rung for the Fe4-Fe2-Fe4 ladders similar to the one represented in figure 5-a), and the same ordering as previously for the Fe3-Fe1-Fe3 ladders. We start from a magnetic moment of $5 \mu_{B}$. This magnetic ordering is preserved during our simulation and the structure of the Fe4-Fe2-Fe4 ladders evolves towards the zigzag structural ordering similar to the Pbnm experimental phase [7] as presented in figure 5-a). Our result shows that, similarly to the double exchange or kinetic exchange mechanism, ferromagnetic bonds are reenforced as compared to antiferromagnetic ones due to an increasing internal hopping. As a consequence, ferromagnetic bonds become shorter hence favoring the structural zigzag ordering.

In our phase triads have a slight zigzag due to the displacement of the central ions. Two different distances were obtained, the short distance is 2.796 Aand the large one $2.885 \AA$, see Table 2. In the Pbnm experimental phase the corresponding distances are (short) 2.616 Aand (large) $2.942 \AA[7]$. This magnetic ordering could be comparable to the $\mathrm{I}_{b}$ phase of reference [16] where the orientation of the magnetic moments along central leg is different from 


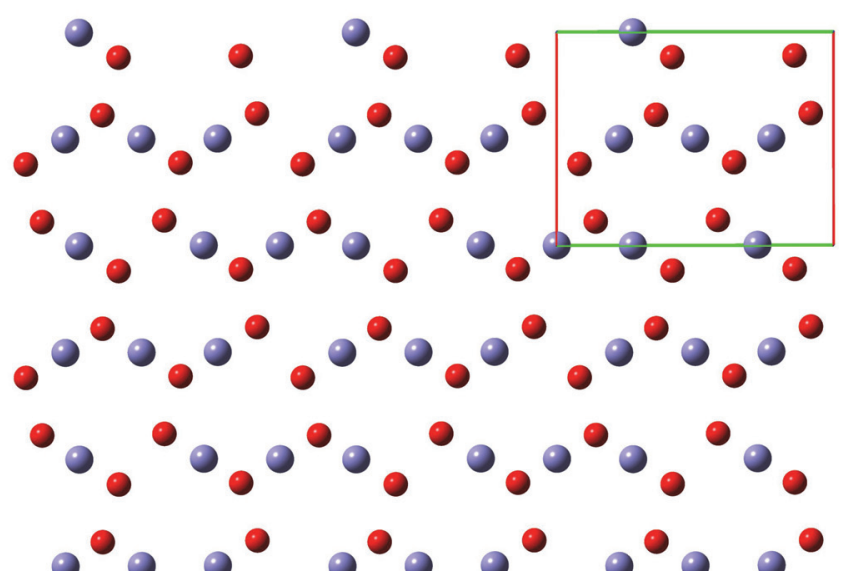

Fig. 4. Perspective in the $\mathrm{a}-\mathrm{b}(\mathrm{x}-\mathrm{y})$ plane at $\mathrm{z}=\mathrm{c} \sim 3.1 \AA$ of the Fe3-Fe1-Fe3 triads (three near horizontal Fe (blue) atoms) of the 1_O structure obtained from Fig. 2. Blue and red colors correspond to $\mathrm{Fe}$ and $\mathrm{O}$ atoms respectively. Rectangle is $\mathrm{a}=9.608 \AA$ (red line) and $b=12.452 \AA$ (green line) unit cell in accord to Table 1, and Fig. 2. Details in text.

$\pi$. Structural, electronic and magnetic correlation was observed and a zigzag structural and magnetic phases were obtained [16].

The magnetic behavior of the $\mathrm{Fe} 4-\mathrm{Fe} 2-\mathrm{Fe} 4$ is quite different from what has been found experimentally at $82 \mathrm{~K}$ [11] , see 5-a),b). As observed before charge distribution is mostly located in the center of the triads. The magnetic moment in the center corresponds to $\sim 3.757$ Bohr magnetons $\left(\mu_{B}\right)$ that is again very near to $4 \mu_{B}$ for high spin $\mathrm{Fe}^{2+}$ ions. In contrast, in the extremes of the ladder a magnetic moment of $4.273 \mu_{B}$ was obtained. The magnetic moments obtained by powder diffraction study [11] are $3.9 \mu_{B}$ (in the center) and $2.74 \mu_{B}$ (in the extremes).

The same structural and magnetic ordering (as obtained before for 1_O and 2_M phases) was observed in Fe3-Fe1Fe3 ladders where a distance Fe3-Fe1 of $3.427 \AA$ was calculated, see Table 2 . It is important to note that distances previously observed were $3.425 \AA\left(1 \_\mathrm{O}\right)$ and $3.426 \AA\left(2 \_\mathrm{M}\right)$. The magnetic behavior of Fe3-Fe1-Fe3 ladders is similar as observed before for 1_O and 2_M phases. In this case noncollinear spin polarised calculations were performed. We do not observe important magnetic moments contribution in other directions as presented in Table 3. To compare with results on this phase, it is important to mention again that the experimental (Pbnm structure) magnetic moments of the Pbnm structure of these ladders are located in a perpendicular direction as compared with Fe4-Fe2-Fe4 magnetic moments direction [11]. The experimental magnetic

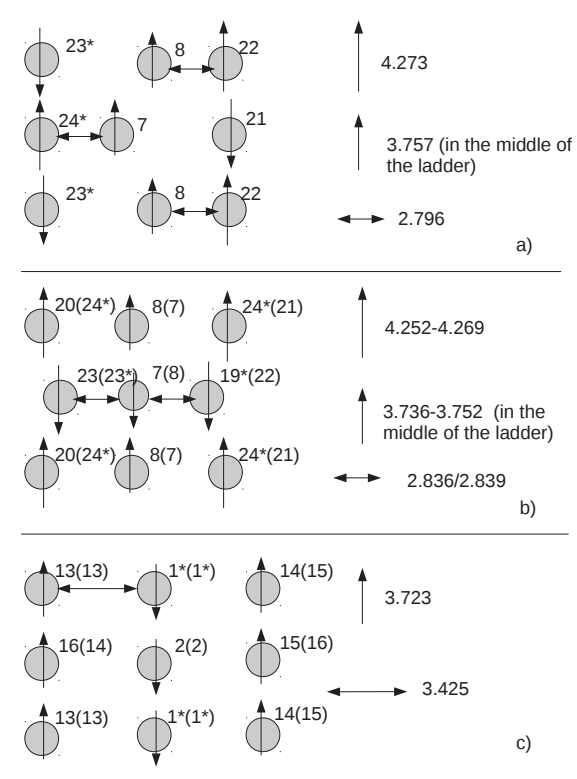

Fig. 5. Picture of the structural and magnetic ordering of the three leg-ladders, triads of Fe atoms of Figs. 3 and 4 along vertical $\mathrm{c}=\mathrm{Z}$ direction. Fe4-Fe2-Fe4 three leg-ladders of the 1_M_NC structure in a), $\mathrm{Fe} 4-\mathrm{Fe} 2-\mathrm{Fe} 4$ three leg-ladders of the 1_O and 2_M structures in b) and Fe3-Fe1-Fe3 three leg-ladders structure for 1_M_NC, 1_O and 2_M in c). Vertical arrows represent magnetic moments in Bohr magnetons. Horizontal lines with two arrows are distances in $\AA$ in accord to Tables 2 and 3. See Figs. 3 and 4 for more reference. Numbering (1_M_NC in a) and 1_O(2_M) in b) and c)) and asterisk (*: crystallographically equivalent atoms) was included to identify atoms in accord to Table 2 and Figs. 1 and 2. Details in text.

moments are $3.2 \mu_{B}$ (in the center) and $4.0 \mu_{B}$ (in the extremes) [11].

\section{Conclusions}

New metastable phases in an oxyborate compound were discussed using an evolutionary algorithm and Density Functional Theory. Our lowest energy monoclinic structure was identified as $\mathrm{P} 21 / \mathrm{m}$ with space group number of 11. An orthorhombic-monoclinic structural transition was observed in this phase where spin-orbit coupling and a particular zigzag magnetic structure were proposed as an explanation of this transition. The structural and magnetic distortion of this monoclinic phase follows a zigzag ordering like the zigzag structural one observed in the low temperature phase of the Fe-homometallic Ludwigite compound. Furthermore a new zigzag structural ordering in three-leg ladders was observed in our new 1_O and 2_M metastable low-energy crystal systems. In the former ordering large and short rungs intercalate in a zigzag way along c direction. Magnetic order in these ladders behave qualitatively similar to the low temperature Pbnm experimental structure where ferromagnetic ordering inside rungs and rungs ordered antiferromagnetically in $\mathrm{c}$ direction was observed. A possible explanation of this particular zigzag behavior (because of rungs distance) could 
be the well known structural instability in low dimensional systems. In this case the symmetry in the ferromagnetic magnetic ordering inside rungs prevents the slight zigzag appearance due to the small displacement of the central ions ( $\mathrm{Fe} 2$ ) as observed in the Pbnm experimental structure giving a natural zigzag distortion in rungs distance. It is found that magnetic symmetry like the one observed in our 1_O and 2_M systems or even in the low temperature Pbnm experimental structure is not favorable for a zigzag structural ordering. This work could be a motivation for more experimental and theoretical work of the homometallic Fe-Ludwigite at low temperature.

\section{ACKNOWLEDGMENT}

The computational simulations of this research were conducted at the Center for Nanophase Materials Sciences (CNMS), which is a DOE Office of Science User Facility. Some of the calculations used resources at the National Energy Research Scientific Computing Center NERSC, a DOE Office of Science User Facility at Oak Ridge National Laboratory (ORNL). One of us E. V. thanks PROMEP UAEHPTC-723 DSA/103.5/15/10450 for financial support.

\section{References}

[1] J. C. Fernandes, R. B. Guimares, M. A. Continentino, H. A. Borges, J. V. Valarelli, and A. Lacerda; Titanium-III warwickites: A family of one-dimensional disordered magnetic systems; Phys. Rev. B 50, 16754(R) (1994).

[2] R. B. Guimarães, J. C. Fernandes, M. A. Continentino, H. A. Borges, C. S. Moura, J. B. M. da Cunha, and C. A. dos Santos; Dimensional crossover in magnetic warwickites; Phys. Rev. B 56, 292 (1997).

[3] M. A. Continentino, A. M. Pedreira, R. B. Guimarães, M. Mir, J. C. Fernandes, R. S. Freitas, and L. Ghivelder; Specific heat and magnetization studies of $\mathrm{Fe}_{2} \mathrm{OBO}_{3}, \mathrm{Mn}_{2} \mathrm{OBO}_{3}$, and $\mathrm{MgScOBO}_{3}$; Phys. Rev. B 64, 014406 (2001).

[4] J. C. Fernandes, R. B. Guimarães, M. A. Continentino, H. A. Borges, A. Sulpice, J-L. Tholence, J. L. Siqueira, L. I. Zawislak, J. B. M. da Cunha, and C. A. dos Santos; Magnetic interactions in the ludwigite $\mathrm{Ni}_{2} \mathrm{FeO}_{2} \mathrm{BO}_{3}$; Phys. Rev. B 58, 287 (1998).

[5] R. B. Guimarães, M. Mir, J. C. Fernandes, M. A. Continentino, H. A. Borges, G. Cernicchiaro, M. B. Fontes, D. R. S. Candela, and E. Baggio-Saitovitch; Cation-mediated interaction and weak ferromagnetism in $\mathrm{Fe}_{3} \mathrm{O}_{2} \mathrm{BO}_{3}$; Phys. Rev. B 60, 6617 (1999).

[6] J. C. Fernandes, R. B. Guimarães, M. A. Continentino, L. Ghivelder, and R. S. Freitas; Specific heat of $\mathrm{Fe}_{3} \mathrm{O}_{2} \mathrm{BO}_{3}$ : Evidence for a Wigner glass phase; Phys. Rev. B 61, R850(R) (2000).

[7] M. Mir, R. B. Guimarães, J. C. Fernandes, M. A. Continentino, A. C. Doriguetto, Y. P. Mascarenhas, J. Ellena, E. E. Castellano, R. S. Freitas, and L. Ghivelder; Structural Transition and Pair Formation in $\mathrm{Fe}_{3} \mathrm{O}_{2} \mathrm{BO}_{3}$; Phys. Rev. Lett. 87, 147201 (2001).

[8] D. C. Freitas, M. A. Continentino, R. B. Guimarães, J. C. Fernandes, E. P. Oliveira, R. E. Santelli, J. Ellena, G. G. Eslava, and L. Ghivelder; Partial magnetic ordering and crystal structure of the ludwigites $\mathrm{Co}_{2} \mathrm{FeO}_{2} \mathrm{BO}_{3}$ and $\mathrm{Ni}_{2} \mathrm{FeO}_{2} \mathrm{BO}_{3}$; Phys. Rev. B 79, 134437 (2009).

[9] J. Bartolomé, A. Arauzo, N. V. Kazak, N. B. Ivanova, S. G. Ovchinnikov, Yu. V. Knyazev, and I. S. Lyubutin; Uniaxial magnetic anisotropy in $\mathrm{Co}_{2.25} \mathrm{Fe}_{0.75} \mathrm{O}_{2} \mathrm{BO}_{3}$ compared to $\mathrm{Co}_{3} \mathrm{O}_{2} \mathrm{BO}_{3}$ and $\mathrm{Fe}_{3} \mathrm{O}_{2} \mathrm{BO}$ ludwigites; Phys. Rev. B 83, 144426 (2011).

[10] N. V. Kazak, N. B. Ivanova, O. A. Bayukov, S. G. Ovchinnikov, A. D. Vasiliev, V. V. Rudenko, J. Bartolomé, A. Arauzo, Yu. $\mathrm{V}$. Knyazev; The superexchange interactions in mixed $\mathrm{Co}-\mathrm{Fe}$ ludwigite; J. Magn. Magn. Mater. 323, 521 (2011).

[11] P. Bordet and E. Suard; Magnetic structure and charge ordering in $\mathrm{Fe}_{3} \mathrm{BO}_{5}$ : A single-crystal $x$-ray and neutron powder diffraction study; Phys. Rev. B 79, 144408 (2009).

[12] J. Larrea, D. R. Sánchez, F. J. Litterst, E. M. Baggio-Saitovitch, J. C. Fernandes, R. B. Guimarães, and M. A. Continentino; Magnetism and charge ordering in $\mathrm{Fe}_{3} \mathrm{O}_{2} \mathrm{BO}_{3}$ studied by ${ }^{57} \mathrm{Fe}$ Mössbauer spectroscopy Phys. Rev. B 70, 174452 (2004).

[13] M. H. Whangbo, H-J Koo, J. Dumas, M. A. Continentino; Theoretical investigation of the spin exchange interactions and magnetic properties of the homometallic ludwigite $\mathrm{Fe}_{3} \mathrm{O}_{2} \mathrm{BO}_{3}$; Inorg. Chem. 41, 2193 (2002).

[14] M. Matos and A. X. Souza; Electronic structure analysis of two crystalline phases of $\mathrm{Fe}_{3} \mathrm{O}_{2} \mathrm{BO}_{3}$; Int. J. Quantum Chem. 103, $562(2005)$.

[15] A. Latgé and M. A. Continentino; Transverse charge density waves in ladder systems; Phys. Rev. B 66, 094113 (2002).

[16] E. Vallejo and M. Avignon; Spin and Charge Ordering in ThreeLeg Ladders in Oxyborates; Phys. Rev. Lett. 97217203 (2006).

[17] M. Matos, J. Terra, D. E. Ellis and A. S. Pimentel; First principles calculation of magnetic order in a low-temperature phase of the iron ludwigite; J. Magn. Magn. Mater. 374, 148 (2015).

[18] D. C. Freitas, M. A. Continentino, R. B. Guimarães, J. C. Fernandes, J. Ellena, and L. Ghivelder; Structure and magnetism of homometallic ludwigites: $\mathrm{Co}_{3} \mathrm{O}_{2} \mathrm{BO}_{3}$ versus $\mathrm{Fe}_{3} \mathrm{O}_{2} \mathrm{BO}_{3}$; Phys. Rev. B 77, 184422 (2008).

[19] E. Vallejo and G. Calderón; Structural distortion in Ludwigites; Mod. Phys. Lett. B 27, 1350131 (2013).

[20] A. R. Oganov and C. W. Glass; Crystal structure prediction using ab initio evolutionary techniques: principles and applications. J. Chem. Phys. 124, 244704 (2006).

[21] A. O. Lyakhov, A. R. Oganov, H. T. Stokes and Q. Zhu; New developments in evolutionary structure prediction algorithm USPEX Comp. Phys. Comm., 184, 1172 (2013).

[22] A. R. Oganov, A. O. Lyakhov and M. Valle; How Evolutionary Crystal Structure Prediction Works-and Why; Acc. Chem. Res., 44(3), 227 (2011).

[23] G. Kresse and J. Hafner; Ab initio molecular dynamics for liquid metals; Phys. Rev. B 47, 558(R) (1993).

[24] G. Kresse and J. Furthmüller; Efficient iterative schemes for ab initio total-energy calculations using a plane-wave basis set; Phys. Rev. B 54, 11169 (1996).

[25] Q. Wang, A. R. Oganov, Q. Zhu, and X-F. Zhou; New Reconstructions of the (110) Surface of Rutile $\mathrm{TiO}_{2}$ Predicted by an Evolutionary Method; Phys. Rev. Lett. 113266101 (2014).

[26] V. Sharma, Ch. Wang, R. G. Lorenzini, R. Ma, Q. Zhu, D. W. Sinkovits, G. Pilania, A. R. Oganov, S. Kumar, G. A. Sotzing, S. A. Boggs and R. Ramprasad; Rational design of all organic polymer dielectrics; Nature Communications 54845 (2014).

[27] E. Vallejo and D. Olguín; New metastable phases in a trititanium pentoxide compound; Mater. Res. Express 2126101 (2015).

[28] P. E. Blöchl; Projector augmented-wave method; Phys. Rev. B 50, 17953 (1994).

[29] G. Kresse and D. Joubert; From ultrasoft pseudopotentials to the projector augmented-wave method; Phys. Rev. B 59, 1758 (1999).

[30] J. P. Perdew, K. Burke and M. Ernzerhof; Generalized Gradient Approximation Made Simple; Phys. Rev. Lett. 77, 3865 (1996).

[31] S. L. Dudarev, G. A. Botton, S. Y. Savrasov, C. J. Humphreys, and A. P. Sutton; Electron-energy-loss spectra and the structural stability of nickel oxide:An LSDA+U study; Phys. Rev. B 57, 1505 (1998). 
[32] H. T. Stokes and D. M. Hatch; FINDSYM: Program for Identifying the Space Group Symmetry of a Crystal; J. Appl. Cryst. 38, 237 (2005). 